\title{
Importância da atuação do farmacêutico clínico nos serviços de diálise: uma revisão narrativa
}

\author{
Importance of clinical pharmacist in dialysis services: a narrative review \\ Importancia del farmacéutico clínico en los servicios de diálisis: una revisión narrativa
}

Flávia Matos e Oliveira ${ }^{1 *}$, Juliana Lima Gomes Rodrigues.

\begin{abstract}
RESUMO
Objetivo: Relatar a importância da atuação do profissional farmacêutico clínico nos serviços de diálise. Revisão bibliográfica: Pacientes com doença renal crônica em diálise apresentam elevado índice de comorbidades e frequentemente utilizam fármacos de várias classes terapêuticas. O tratamento implica um grande arsenal de prescrições e complexos regimes terapêuticos que elevam o risco de eventos adversos, interações medicamentosas e outros problemas relacionados a medicamentos. A revisão foi organizada nos seguintes tópicos: epidemiologia da doença renal crônica, perfil de pacientes, complexidade do tratamento medicamentoso na doença renal crônica, importância da conciliação medicamentosa e oportunidades de atuação do farmacêutico clínico nos serviços de diálise, assim como as possíveis barreiras para sua implementação. Considerações finais: Através das evidências apresentadas, foi possível chamar a atenção para as diversas formas de contribuição do farmacêutico clínico para a melhoria do processo de uso dos medicamentos, a redução dos riscos, o aprimoramento da qualidade dos serviços prestados e melhoria de desfechos dos pacientes em diálise.
\end{abstract}

Palavras-chave: Farmacêutico, Hemodiálise, Assistência farmacêutica, Doença renal crônica.

\begin{abstract}
Objective: To report the importance of the role of the clinical pharmacist in dialysis services. Literature review: Patients with chronic kidney disease on dialysis have a high rate of comorbidities and frequently use drugs from different therapeutic classes. Treatment involves a large arsenal of prescriptions and complex therapeutic regimens that increase the risk of adverse events, drug interactions and other drug-related problems. The review was organized into the following topics: epidemiology of chronic kidney disease, patient profile, complexity of drug treatment in chronic kidney disease, importance of medication reconciliation and opportunities for clinical pharmacist to act in dialysis services, as well as possible barriers to its Implementation. Final considerations: Through the evidence presented, it was possible to call attention to the several ways in which the clinical pharmacist can contribute to improving the process of medication use, reducing risks, improving the quality of services provided and improving patient outcomes on dialysis.
\end{abstract}

Key words: Pharmacist, Hemodialysis, Pharmaceutical assistance, Chronic kidney disease.

\section{RESUMEN}

Objetivo: Informar la importancia del papel del farmacéutico clínico en los servicios de diálisis. Revisión de la literatura: Los pacientes con enfermedad renal crónica en diálisis tienen una alta tasa de comorbilidades y utilizan con frecuencia fármacos de diversas clases terapéuticas. El tratamiento implica un gran arsenal de recetas y regímenes terapéuticos complejos que aumentan el riesgo de eventos adversos, interacciones medicamentosas y otros problemas relacionados con los medicamentos. La revisión se organizó en los siguientes temas: epidemiología de la enfermedad renal crónica, perfil del paciente, complejidad del tratamiento farmacológico en la enfermedad renal crónica, importancia de la conciliación de la medicación y oportunidades para que el farmacéutico clínico actúe en los servicios de diálisis, así como posibles barreras para su implementación. Consideraciones finales: A través de la evidencia presentada, fue posible llamar la atención sobre las diversas formas en que el farmacéutico clínico puede contribuir a mejorar el proceso de uso de medicamentos, reducir riesgos, mejorar la calidad de los servicios prestados y mejorar los resultados de los pacientes en diálisis.

Palabras clave: Farmacéutico, Hemodiálisis, Asistencia farmacéutica, Enfermedad renal crónica.

${ }^{1}$ Universidade Salvador, Salvador - BA. *E-mail: fmo.farma@gmail.com 


\section{INTRODUÇÃO}

A Doença Renal Crônica (DRC) é hoje um problema de saúde pública em todo mundo. Causada por agressões persistentes e irreversíveis ao parênquima renal que levam a redução progressiva da taxa de filtração glomerular, caracteriza-se clinicamente pela elevação de escórias nitrogenadas, acidose metabólica, alterações eletrolíticas, entre outras anormalidades (BRASIL, 2014).

Quando a doença renal atinge seu estágio mais avançado, a falência renal resulta na necessidade de se iniciar a Terapia Renal Substitutiva (TRS), cujas opções disponíveis são a hemodiálise, a diálise peritoneal e o transplante renal. Embora o transplante seja considerado a melhor opção, a hemodiálise é a terapia mais utilizada em nível mundial. Além da TRS, os pacientes devem aderir ao tratamento dietético e farmacológico (BRASIL, 2014).

Segundo o Inquérito Anual de Diálise Crônica de 2019 da Sociedade Brasileira de Nefrologia (SBN), a prevalência de pacientes em diálise no Brasil alcançou a taxa de 684 pacientes por milhão de população, 0 que representa um aumento de $30 \%$ nos últimos 5 anos, perfazendo o total de 144.779 pacientes em 2019 . (SBN, 2020).

Em relação ao perfil dos pacientes em diálise no Brasil, observou-se um grande percentual de idosos, com $36 \%$ dos pacientes maiores de 64 anos e $58 \%$ do sexo masculino. As causas primárias mais frequentes da DRC dialítica foram Hipertensão Arterial Sistêmica (HAS), em 32\% dos pacientes, e diabetes, em $31 \%$. A taxa de mortalidade bruta estimada foi de 18\%. O Sistema Único de Saúde (SUS) é a principal fonte pagadora, sendo responsável pelo financiamento de $79 \%$ dos pacientes em tratamento dialítico (SBN, 2020).

Além da alta prevalência do diabetes e da hipertensão arterial, pacientes em tratamento dialítico apresentam elevado índice de comorbidades, geralmente relacionadas a complicações cardiovasculares, anemia, alterações do metabolismo mineral, doença osséa, desnutrição, entre outras. Consequentemente utilizam fármacos de várias classes terapêuticas, incluindo anti-hipertensivos, antidiabéticos, hipolipemiantes, quelantes de fósforo, análogos da vitamina $\mathrm{D}$, agentes estimuladores da eritropoiese, compostos de ferro, entre outros (BRASIL, 2014).

Por serem polimedicados, estes pacientes apresentam alto risco para Problemas Relacionados a Medicamentos (PRM) (ALSHAMRANI M, et al., 2018). A identificação das fragilidades no processo de uso de medicamentos e possíveis intervenções por parte do farmacêutico podem contribuir para melhorar a segurança medicamentosa, melhorar desfechos e reduzir o custo do tratamento (LOCATELLI C, et al., 2015).

A Resolução da Diretoria Colegiada (RDC) № 11 de 13 de março de 2014 da Agência Nacional de Vigilância Sanitária (ANVISA), que dispõe sobre o funcionamento de serviços de diálise no Brasil, não contempla a atuação do farmacêutico clínico na equipe multidisciplinar de assistência ao paciente renal. Segundo esta RDC, a atuação deste profissional está restrita à supervisão da fabricação e controle da qualidade do concentrado polieletrolítico para o banho de diálise (ANVISA, 2014). Entretanto, diversos estudos trazem evidências que suportam a inclusão do referido profissional na equipe assistencial dos serviços de diálise, contribuindo de diversas formas na melhoria da qualidade do cuidado e dos desfechos no tratamento destes pacientes (KIMURA T, et al., 2004; PAI AB, et al., 2009a; SALGADO TM, et al., 2012a; SALGADO TM, et al., 2013 e TESFAYE WH, et al., 2017).

O objetivo deste trabalho foi realizar uma revisão narrativa da literatura sobre a importância da atuação do profissional farmacêutico clínico nos serviços de hemodiálise.

\section{REVISÃO BIBLIOGRÁFICA}

\section{Complexidade da farmacoterapia na doença renal crônica}

Entender a complexidade do tratamento medicamentoso de pacientes em diálise é relevante para compreender a importância da atuação do farmacêutico clínico junto à equipe multidisciplinar nos serviços de diálise. Voltado para as doenças de base ou para as anormalidades decorrentes da falência renal, 0 
tratamento implica um grande arsenal de prescrições e complexos regimes terapêuticos que elevam o risco de eventos adversos, Interações Medicamentosas (IM) e outros PRM (SPANEVELLO S, et al., 2018).

\section{Tratamento da hipertensão e complicações cardiovasculares}

A hipertensão afeta até $90 \%$ da população de pacientes em hemodiálise e é um fator de risco para desfechos cardiovasculares adversos. O primeiro passo no tratamento da hipertensão em pacientes em diálise é o controle do volume do fluido extracelular através da manutenção do peso seco na diálise, redução da ingestão de sal e restrição hídrica (ALMEIDA FA, et al., 2002). Entretanto a administração de antihipertensivos também é necessária para o controle dos valores pressóricos nesta população.

A maioria das classes destes medicamentos é cabível para uso nestes pacientes, sendo comum a prescrição de vários medicamentos anti-hipertensivos para se alcançar o controle efetivo da pressão arterial. Os agentes anti-hipertensivos de primeira linha incluem Inibidores da Enzima de Conversão da Angiotensina (IECA) e Bloqueadores de Receptores de Angiotensina (BRA) (BRASIL, 2014). Simpaticolíticos, betabloqueadores, bloqueadores dos canais de cálcio e vasodilatadores diretos també $m$ são eficazes no controle da pressão arterial destes pacientes. Além dos anti-hipertensivos, são comumente utilizados antiagregantes plaquetários, anticoagulantes, estatinas, digitálicos e antiarrítmicos (ALMEIDA FA, et al., 2002).

\section{Tratamento da anemia}

A anemia é uma complicação frequente em pacientes tratados por diálise. Esta condição decorre da diminuição de produção de eritropoetina pelas células endoteliais nas proximidades dos túbulos renais associada à deficiência de ferro, em decorrência da perda crônica de sangue e diminuição na absorção de ferro da dieta. Portanto, o tratamento da anemia na DRC consiste na reposição de compostos de ferro e de eritropoetina. A administração deste último, pode ser feita por via endovenosa ou subcutânea, já a administração de ferro se faz preferencialmente por via endovenosa, durante a sessão de hemodiálise (BRASIL, 2017).

\section{Distúrbios do metabolismo ósseo e mineral}

Com o declínio da função renal, ocorrem alterações progressivas no metabolismo mineral e ósseo do paciente, prejudicando a homeostase do cálcio, fósforo e dos hormônios reguladores: hormônio da paratireoide, 1,25-hidroxivitamina D e fator de crescimento de fibroblastos-23. A hiperfosfatemia, caracterizada pelo aumento da concentração plasmática de fosfato, em associação com o cálcio, pode levar à calcificação das artérias coronárias e complicações cardiovasculares (CUSTÓDIO MR, et al., 2013).

Para o tratamento dos distúrbios do metabolismo mineral e ósseo, além das medidas dietéticas de restrição de fósforo e adequação da dose de diálise, recomenda-se o uso de quelantes de fósforo (quelantes à base de cálcio ou cloridrato de sevelâmer) e medicamentos para controle do hiperparatireoidismo, como os análogos da vitamina $D$ e calcimiméticos. Os critérios de uso destes fármacos estão estabelecidos no Protocolo Clínico e Diretrizes Terapêuticas Distúrbio Mineral e Ósseo na DRC (CUSTÓDIO MR, et al., 2013).

Assim sendo, a polifarmácia é altamente prevalente nos pacientes em diálise cujo número médio de medicamentos em uso pode variar de 5 a 14 por dia, com uma média de 17 a 25 doses diárias (PAI AB, et al., 2013 e TESFAYE WH, et al., 2017).

\section{Potenciais Interações Medicamentosas na Farmacoterapia dos Pacientes em Diálise}

A prevalência de IM em pacientes em diálise é elevada. Um estudo realizado a partir da análise de prescrições médicas de pacientes nefrológicos de um hospital de referência do nordeste brasileiro demonstrou que $83 \%$ das prescrições apresentavam interações medicamentosas. A maioria das interações foram consideradas de menor gravidade, entretanto cerca de $12 \%$ dos casos foram classificados como de maior gravidade, como a coadministração de anlodipino e sinvastatina. Esta associação aumenta o risco de ocorrência de miopatias, incluindo rabdomiólise, pois o anlodipino aumenta os níveis séricos da estatina através de modificações em sua farmacocinética. Também chamou a atenção a elevada ocorrência da 
prescrição de atenolol com clonidina, associação que aumenta o risco de hipertensão rebote (NEIVA LDB, et al., 2019).

Para prevenir IM, os responsáveis pelas prescrições de medicamentos em pacientes com DRC devem estar cientes dos fatores determinantes da sua ocorrência e especialmente atentos aos possíveis efeitos da insuficiência renal sobre a excreção renal de fármacos. Castelino RL, et al. (2011) chamam atenção para a possibilidade de alterações no metabolismo e eliminação dos fármacos em decorrência da DRC, assim como os possíveis efeitos da diálise sobre a farmacocinética dos medicamentos, sendo então de suma importância que se atente para o monitoramento e ajuste das doses.

É interessante ainda ressaltar a frequente falta de informações sobre a duração do tratamento e precárias recomendações de uso, uma vez que tais itens podem resultar em tempo e forma de uso inadequados, aumentando os riscos de ocorrência de efeitos não desejados, como a intoxicação medicamentosa, interações e reações adversas a medicamento (LEÃO DFL, et al., 2014).

Portanto, é fundamental avaliar e monitorar IM uma vez que estas podem agravar a condição clínica do paciente. Para esse monitoramento, faz-se necessária a atuação da equipe multidisciplinar, com destaque para a atuação do farmacêutico, que pode fornecer orientações em prol de um manejo terapêutico adequado e eventualmente sugerir a substituição de medicamentos que possam agravar o dano renal (SPANEVELLO S, et al., 2018).

\section{Oportunidades de atuação do farmacêutico clínico em serviços de diálise}

Existem evidências de que o envolvimento do farmacêutico clínico na linha de cuidado do paciente com DRC pode melhorar desfechos e reduzir custos para o sistema de saúde (KIMURA T, et al., 2004; PAI AB, et al., 2009a; SALGADO TM, et al., 2012a; SALGADO TM, et al., 2013 e TESFAYE WH, et al., 2017).

Uma revisão sistemática sobre prescrições inapropriadas em pacientes com DRC mostrou que o feedback imediato de um farmacêutico clínico para o médico prescritor foi o tipo de intervenção que mais reduziu a ocorrência de prescrições inapropriadas (TESFAYE WH, et al., 2017).

Kimura T, et al. (2004) constataram maior proporção de pacientes que alcançaram meta de hemoglobina em protocolos de manejo de anemia conduzidos por farmacêutico. Um outro estudo no qual farmacêuticos tinham autonomia para ajustar as doses de quelantes de fósforo e análogos de vitamina $D$ de pacientes com DRC resultou na redução significativa no número de pacientes com hiperparatireoidismo secundário moderado e grave (SALGADO TM, et al., 2012a).

Redução das taxas de hospitalização, redução no número de medicamentos utilizados e melhora geral na qualidade de vida dos pacientes em diálise também têm sido reportadas como resultados de intervenções do farmacêutico junto à equipe assistencial (PAI AB, et al., 2009a; PAI AB, et al., 2009b).

A não adesão ao tratamento farmacológico é um problema comum nos pacientes em diálise, e a falta de compreensão das instruções de uso dos medicamentos é uma causa importante para a não adesão medicamentosa. $O$ farmacêutico pode assegurar que o paciente tenha e entenda a informação necessária para utilizar o medicamento (AMARANTE LC, et al., 2010; NERBASS FB, et al., 2010) .

Portanto as possibilidades de atuação do farmacêutico em unidades de diálise incluem desde funções técnicas, nas quais ele garante todos os requisitos técnicos e legais do ciclo da assistência farmacêutica, até funções clínicas, onde pode desempenhar intervenções centradas no paciente, como prover informações relacionadas aos medicamentos a este e à equipe multidisciplinar (VIANA ALS, 2017).

A resolução n 672 de 2019 do Conselho Federal de Farmácia (CFF) dispõe sobre a atuação do farmacêutico em serviços de diálise (CFF, 2019). As atribuições clínicas deste profissional em serviços de diálise segundo esta resolução estão descritas abaixo (Quadro 1). 
Quadro 1 - Atribuições clínicas do farmacêutico em serviços de diálise segundo Resolução no 672/2019 do Conselho Federal de Farmácia.

\section{Atribuições Clínicas do Farmacêutico em Serviços De Diálise}

a) Prevenir, identificar, avaliar, monitorar e intervir nos incidentes relacionados aos medicamentos e a outros problemas relacionados a farmacoterapia e demais produtos utilizados na assistência ao paciente;

b) Acessar, conhecer, interpretar e organizar as informações constantes do prontuário, a fim de proceder à avaliação do paciente;

c) Conhecer as condições fisiopatológicas do paciente;

d) Fazer a anamnese farmacêutica, incluindo a história da doença atual, comorbidades, hábitos de vida, alergias conhecidas, uso prévio de medicamentos, entre outros;

e) Fazer a conciliação de medicamentos;

f) Fazer o acompanhamento farmacoterapêutico;

g) Analisar a prescrição do paciente quanto aos aspectos legais e técnicos, de modo a promover o uso adequado de medicamentos e de outros produtos para a saúde;

h) Avaliar a farmacoterapia quanto à indicação, doses, frequência, horários, vias de administração, formas farmacêuticas, dialisabilidade, reconstituição, diluição, tempo e velocidade de infusão, duração do tratamento, com o objetivo de atender às necessidades individuais do paciente;

i) Gerenciar, avaliar e otimizar a terapia antimicrobiana, com o objetivo de promover o uso racional de antimicrobianos, em consonância com as diretrizes da instituição relacionadas ao controle de infecção;

l) Monitorar a necessidade de ajuste de dose de acordo com as características do fármaco, função renal e sistema de diálise.

Fonte: Oliveira FM e Rodrigues JLG, 2021; dados extraídos do CFF, 2019a.

Apesar das crescentes evidências que suportam os benefícios da atuação do farmacêutico clínico em serviços de diálise, barreiras para sua implementação na rotina de funcionamento destes serviços precisam ser consideradas: a necessidade de qualificação dos profissionais farmacêuticos, definição de protocolos bem estabelecidos para sua atuação, eventuais barreiras organizacionais internas como resistências por parte de médicos e enfermeiras e a importância do suporte financeiro para viabilizar a inclusão deste profissional na equipe assistencial dos serviços de diálise (SALGADO TM, et al., 2012 b; TESFAYE WH, et al., 2017).

No caso do Brasil, embora exista uma resolução do Conselho Federal de Farmácia, definindo claramente o papel do farmacêutico clínico nos serviços de diálise, a Sociedade Brasileira de Nefrologia defende a ideia de que cada serviço tenha autonomia para dimensionar sua equipe assistencial de acordo com a realidade local. Associação Brasileira de Clinica de Dialise e Transplante (ABCDT), por sua vez, chamou atenção para a escassez de farmacêuticos clínicos especializados em diversas regiões e alegou problemas de financiamento dentro do atual contexto da remuneração do SUS para os procedimentos de diálise, manifestando-se contrária à regulamentação do CFF (ABCDT, 2019).

O CFF repudiou veementemente este posicionamento e reafirmou os impactos positivos da atuação do profissional farmacêutico nestes serviços, como a possibilidade da redução da mortalidade dos custos econômicos associados a má utilização dos medicamentos nos serviços de diálise (CFF, 2019b).

A atuação clínica do farmacêutico junto ao paciente com doença renal crônica tem se consolidado em vários países, desde o tratamento conservador até a terapia renal substitutiva. Portugal, Estados Unidos, Canadá, Reino Unido, Nova Zelândia e Austrália são exemplos de países que já incluíram o farmacêutico na equipe assistencial ao paciente renal (ST. PETER WL, 2013 e VIANAALS, 2017). A inclusão desse profissional nos serviços de nefrologia e TRS tem como objetivo melhorar a segurança e otimizar o tratamento farmacológico destes pacientes, contribuindo para melhoria da qualidade dos serviços prestados a estes indivíduos e redução dos riscos.

\section{Importância da conciliação medicamentosa em unidades de diálise}

Entre as ações voltadas para a segurança do paciente, destaca-se o processo de Conciliação Medicamentosa (CM). A CM é um processo formal no qual obtém-se uma lista completa, precisa e atualizada 
dos medicamentos que cada paciente utiliza (incluindo nome, dose, frequência e via de administração), que deve ser comparada com as prescrições médicas feitas em momentos de transição da assistência (admissão, transferência, consultas ambulatoriais ou alta hospitalar). Essa lista é usada para aperfeiçoar a utilização dos medicamentos em todos os pontos de transição do cuidado e tem como principal objetivo diminuir problemas relacionados com medicamentos (SÁNCHEZ OD, et al., 2007).

A partir da CM é possível identificar as discrepâncias medicamentosas, sendo estas definidas como qualquer diferença encontrada entre o histórico de medicamentos utilizado pelo paciente e os medicamentos prescritos para este na admissão hospitalar ou mesmo nos tratamentos ambulatoriais (PAI AB, et al., 2013). Se forem encontradas discrepâncias, estas devem ser discutidas com o médico e, se apropriado, a prescrição deve ser ajustada. As alterações de prescrição ocorrem principalmente nos esquemas terapêuticos de pacientes após hospitalização, transferidos de outras unidades ou que recorrem a outras especialidades médicas (VIANA ALS, 2017).

Em serviços de diálise, além da necessidade da CM quando da mudança do nível de assistência do paciente, este processo é importante para harmonizar a prescrição ambulatorial feita pelo nefrologista com os medicamentos prescritos por médicos de outras especialidades. Devido às múltiplas comorbidades, pacientes em diálise podem ter vários prescritores envolvidos no seu tratamento com frequentes mudanças em sua prescrição. A CM contribui para 1. manter a lista de medicamentos de cada paciente atualizada, assegurando a continuidade terapêutica de forma racional e segura, 2. compatibilizar alterações da medicação, 3. prevenir erros relacionados com os medicamentos, como duplicação e interações medicamentosas, além de 4. detectar possíveis alergias e contraindicações, visto que, devido à condição da sua doença, os pacientes com DRC apresentam uma vasta quantidade de medicamentos contraindicados (VIANA ALS, 2017).

\section{CONSIDERAÇÕES FINAIS}

Neste artigo evidenciamos a importância do farmacêutico clínico no contexto da doença renal crônica, especialmente em serviços de diálise, onde os pacientes apresentam múltiplas comorbidades e são comumente submetidos à polifarmácia. Olhar clínico do farmacêutico é de suma importância, visando a melhorar os desfechos da terapêutica. Este profissional pode contribuir de diversas formas para a melhoria do processo de uso dos medicamentos, redução dos riscos, aprimoramento da qualidade dos serviços prestados aos pacientes e redução de custos para os sistemas de saúde.

\section{REFERÊNCIAS}

1. ALMEIDA FA, et al. Tratamento da hipertensão arterial no paciente com deficit de função renal. Revista Brasileira de Hipertensão, 2002; 9(3): 288-292.

2. ALSHAMRANI M, et al. Polypharmacy and medication-related problems in hemodialysis patients: a call for deprescribing. Pharmacy, 2018; 6(3): 76.

3. AMARANTE LC, et al. A influência do acompanhamento farmacoterapêutico na adesão à terapia anti-hipertensiva e no grau de satisfação do paciente. Revista de Ciências Farmacêuticas Básica e Aplicada, 2010; 31(3): $209-215$.

4. ASSOCIAÇÃO BRASILEIRA DE CLINICA DE DIALISE E TRANSPLANTE (ABCDT). ABCDT diverge dos conselhos de enfermagem e farmárcia sobre ampliação de profissionais nas clínicas de diálise. 2019. Disponível em: https://www.abcdt.org.br/2019/11/abcdt-diverge-dos-conselhos-de-enfermagem-e-farmarcia-sobre-ampliacao-deprofissionais-nas-clinicas-de-dialise/. Acesso em: 28 abril 2021.

5. AGÊNCIA NACIONAL DE VIGILÂNCIA SANITÁRIA (ANVISA). Resolução - RDC n 11, de 13 de março de 2014. Dispõe sobre os requisitos de boas práticas de funcionamento para os serviços de diálise e dá outras providências. Disponivel

em: http://www.rio.rj.gov.br/dlstatic/10112/5125745/4208567/RDCANVISAn11de13_03_2014boaspraticasdefuncioname ntoparaosservicosdedialise.pdf. Acessado em: 10 de maio de 2021.

6. BRASIL. Ministério da Saúde. Diretrizes clínicas para o cuidado ao paciente com doença renal crônica no Sistema

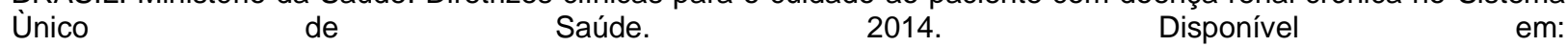
https://bvsms.saude.gov.br/bvs/publicacoes/diretrizes_clinicas_cuidado_paciente_renal.pdf. Acesso em: 28 abril 2021. 
7. BRASIL. Ministério da Saúde. Protocolo clínico e diretrizes terapêuticas anemia na doença renal crônica Alfaepoetina. 2017. Disponivel em: http://portalarquivos.saude.gov.br/images/pdf/2017/marco/06/Protocolo-Cl--nicoE-Diretrizes-Terap--uticas---DRC---Alfaepoetina.pdf. Acesso em: 28 abril 2021.

8. CONSELHO FEDERAL DE FARMÁCIA (CFF). Resolução n 672, de 18 de setembro de 2019 - DOU. 2019 a. Disponível em: https://www.in.gov.br/en/web/dou/-/resolucao-n-672-de-18-de-setembro-de-2019-218530503. Acesso em: 27 abril 2021

9. CONSELHO FEDERAL DE FARMÁCIA (CFF). Em defesa da qualidade dos serviços de diálise no Brasil. $2019 \mathrm{~b}$. Disponível

em: https://www.cff.org.br/noticia.php?id=5559\&titulo=EM+DEFESA+DA+QUALIDADE+DOS+SERVIÇOS+DE+DIÁLISE +NO+BRASIL. Acesso em: 6 de abril 2021.

10. CONSELHO FEDERAL DE FARMÁCIA (CFF). Resolução n 672, de 18 de setembro de 2019 - DOU. $2019 a$. Disponível em: https://www.in.gov.br/en/web/dou/-/resolucao-n-672-de-18-de-setembro-de-2019-218530503. Acesso em: 27 abril 2021.

11. CASTELINO RL, et al. Prevalence of medication-related problems among patients with renal compromise in an Indian hospital. Journal of Clinical Pharmacy and Therapeutics. 2011; 36(4): 481-487

12. CUSTÓDIO MR, et al. Protocolo clínico e diretrizes terapêuticas para o tratamento do hiperparatireoidismo secundário em pacientes com doença renal crônica. Jornal Brasileiro de Nefrologia, 2013; 35(4): 308-322.

13. KIMURA T, et al. Impact of a pharmacist-implemented anemia management in outpatients with end-stage renal disease in Japan. Biological and Pharmaceutical Bulletin, 2004; 27(11): 1831-1833.

14. LEÃO DFL, et al. Avaliação de interações medicamentosas potenciais em prescrições da atenção primária de Vitória da Conquista (BA), Brasil. Ciência e Saùde Coletiva; 2014; 19(1): 311-318.

15. LOCATELLI C, et al. Perfil medicamentoso de pacientes sob tratamento de terapia renal substitutiva em um Hospital do Rio Grande do Sul. Revista da Sociedade de Clínica Médica, 2015; 13(4):240-245

16. NEIVA LDB, et al. Interações medicamentosas em pacientes nefrológicos em um hospital de referência do nordeste brasileiro. Revista Eletrônica Acervo Saúde, 2019; (29).

17. NERBASS FB, et al. Adesão e conhecimento sobre o tratamento da hiperfosfatemia de pacientes hiperfosfatêmicos em hemodiálise. Jornal Brasileiro de Nefrologia, 2010; 32(2): 149-155.

18. PAI AB, et al. Health-related quality of life is maintained in hemodialysis patients receiving pharmaceutical care: $A 2-$ year randomized, controlled study. Hemodialysis International, 2009a; 13(1): 72-79.

19. PAI AB, et al. Medication reconciliation and therapy management in dialysis-dependent patients: Need for a systematic approach. Clinical Journal of the American Society of Nephrology, 2013; 8(11), 1988-1999.

20. PAl $A B$, et al. Reduced drug use and hospitalization rates in patients undergoing hemodialysis who received pharmaceutical care: A 2-year, randomized, controlled study. Pharmacotherapy, 2009b; 29(12):1433-1440,

21. RAYMOND CB, et al. Standards of clinical practice for renal pharmacists. Canadian Journal of Hospital Pharmacy, 2013; 66(6): 369-374.

22. SALGADO TM, et al. Assessing the implementability of clinical pharmacist interventions in patients with chronic kidney disease: an analysis of systematic reviews. Annals of Pharmacotherapy, 2013; 47(11): 1498-1506.

23. SALGADO TM, et al. Pharmacists' interventions in the management of patients with chronic kidney disease: $A$ systematic review. Nephrology Dialysis Transplantation, 2012a; 27(1):276-292.

24. SALGADO TM, et al. Exploring the role of renal pharmacists in outpatient dialysis centres: a qualitative study. International Journal of Clinical Pharmacy, 2012b; 34(4):569-78.

25. SÁNCHES OD, et al. Conciliación de la medicación. Medicina Clínica, 2007; 129(9): 343-348.

26. SOCIEDADE BRASILEIRA DE NEFROLOGIA. Inquérito Anual de Diálise Crônica de 2019. 2020.

27. SPANEVELLO S, et al. Interações medicamentosas, reações adversas e ajuste de dose de medicamentos utilizados por pacientes em hemodiálise. Revista Saúde (Sta. Maria), 2018; 44(3).

28. ST. PETER WL, et al. New models of chronic kidney disease care including pharmacists: Improving medication reconciliation and medication management. Current Opinion in Nephrology and Hypertension, 2013; 22(6): 656-662. Disponível em

29. TESFAYE WH, et al. Inappropriate prescribing in chronic kidney disease: A systematic review of prevalence, associated clinical outcomes and impact of interventions. International Journal of Clinical Practice, 2017; 71( 7): 1-16.

30. VIANA ALS. Hemodiálise e o papel do farmacêutico the role of the pharmacist in hemodialysis. Revista Portuguesa de Farmacoterapia, 2017; 9: 107-111. 